\title{
Application of Gaussian Optical Tweezers for Ultrafast Laser Assisted Direct-write Nanostructuring
}

\author{
Ulf QUENTIN $^{1,2}$, Karl-Heinz LEITZ ${ }^{1,2}$, Ilya ALEXEEV ${ }^{1,2}$, Michael SCHMIDT ${ }^{1,2}$ \\ ${ }^{1}$ Chair of Photonic Technologies, University of Erlangen-Nuremberg, Paul-Gordan-Straße 3, \\ 91052 Erlangen, Germany \\ ${ }^{2}$ Erlangen Graduate School in Advanced Optical Technologies, Paul-Gordan-Straße 3, \\ 91052 Erlangen, Germany \\ E-mail: ulf.quentin@lpt.uni-erlangen.de
}

\begin{abstract}
The minimal size of optically generated structures is always affected by diffraction. This fundamental limitation does not allow laser radiation to be focussed much smaller than half of its wavelength and therefore it also limits minimal feature sizes of laser produced structures. In nearfield optics, however, the diffraction limit does not apply, which in principal allows for tighter focussing. In this contribution, a flexible approach for direct-write nanostructuring is presented. Dielectric micro-particles are positioned by Gaussian optical tweezers and irradiated by ultrafast laser pulses. The particles focus the pulses and enable surface structuring.

DOI:10.2961/jlmn.2012.02.0003
\end{abstract}

Keywords: nanostructuring, direct-write, optical trap, ultrafast lasers

\section{Introduction}

The generation of surface structures on a micro- and nanometre scale is of increasing interest for different technical and biomedical applications [1-3]. For the production of such small features, different techniques have been developed and applied in the last decades, such as photolithography, focused ion beam structuring, nanoimprint lithography and laser ablation. Among those, laser based methods are very promising for being contactless and offering high precision. Focussing of a laser beam, however, is affected by diffraction and the smallest achievable focal spot sizes are on the order of half of the laser wavelength [4,5]. This fundamental restriction directly limits the minimal feature size for laser based surface structuring. However, two general approaches have been demonstrated, which allow for sub-diffraction-limited optical material processing: one is to utilize threshold processes and the other one uses near-field effects to overcome the diffraction limit.

\subsection{Optical near-field and threshold effects}

The intensity across a focused laser spot usually possesses a rotational symmetric Gaussian distribution. If the laser power is low enough, the intensity on the substrate exceeds the ablation or modification threshold of the material only in the centre of the Gaussian focal spot. This can be used to generate structures that are smaller than the actual laser spot diameter (see fig. 1). In principle, this works for all laser material combinations, but the effect only becomes significant if there is a sufficiently steep intensity gradient across the laser spot and a pronounced threshold in the material response. The first prerequisite is fulfilled optimally by ultrafast lasers. Due to their short pulse duration and the corresponding high pulse power, ultrashort laser pulses can lead to extremely high intensities of more than $10^{17} \mathrm{~W} / \mathrm{m}^{2}$ and thus to very steep intensity gradients. The requirement of a pronounced threshold can be fulfilled best with materials that normally do not absorb the used laser wavelength but rather need a higher photon energy for processing. High intensities, which mean high photon densities can then leads to multiphoton absorption, which only occur in the centre of the laser spot (see fig. 1). One of the best known processes that utilize threshold effects to generate feature well below the diffraction limit is the two-photon polymerisation [6]. (a)

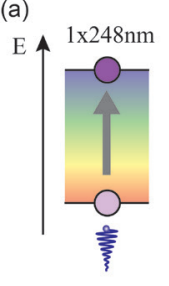

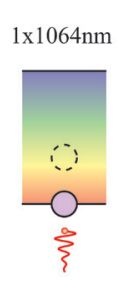

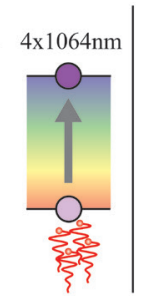

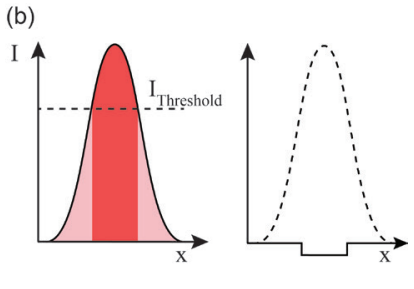

Fig. 1: Simultaneous absorption of multiple photons with the total energy exceeding the bandgap (a). Threshold effects can lead to generated structures that are smaller than the focal spot of the laser (b).

The second possibility to overcome the diffraction limit is the use of near-field effects. Optical near-fields are local electromagnetic field enhancements which decay exponentially, thus do not propagate farther then some tens or hundreds of nanometres [7]. Near-fields arise from the illumination of small particles or apertures, or when an electromagnetic wave passes a step in optical density, e.g. at the crossing of a glass-air-interface [7]. They are not affected by diffraction and can be used to focus laser radiation much smaller than the laser wavelength. This is used in near-field microscopy to enhance the resolution of 
optical imaging systems [8,9], but has also been demonstrated for material processing.

By illuminating the tip of an atomic force microscope (AFM) or light passing through the fibre of a near-field scanning optical microscope (NSOM), optical near-fields are induced, which can be used to modify a substrate's surface with features well below the diffraction limit [10 14]. This process is suitable to generate features with a lateral resolution of $10 \mathrm{~nm}$ [12], but it requires a precise positioning control and thus complex closed loop feedback systems. Furthermore, AFM tips are highly prone to mechanical damage and very expensive.

Another approach to utilize optical near-fields for surface structuring uses spherical micro-particles as nearfield lenses. The microspheres are usually diluted in water and the aqueous solution is coated onto a substrate. As the solvent evaporates, the particles self-organise in hexagonal patterns. Irradiation with laser pulses then leads to nearfield focussing by the particles, which creates subwavelength nano-holes underneath each illuminated particle [14-19]. This process is often referred to as Contacting Particle Lens Array Patterning (CPLA). It is very robust and allows larger areas to be structured, but achievable structures are limited to restricted patterns such as hexagonal patterns. The process depends mostly on the particle material and size, the pulse duration and the substrate material. It has been shown to work on dielectrics, metals and semiconductors with both metallic and dielectric particles [19].

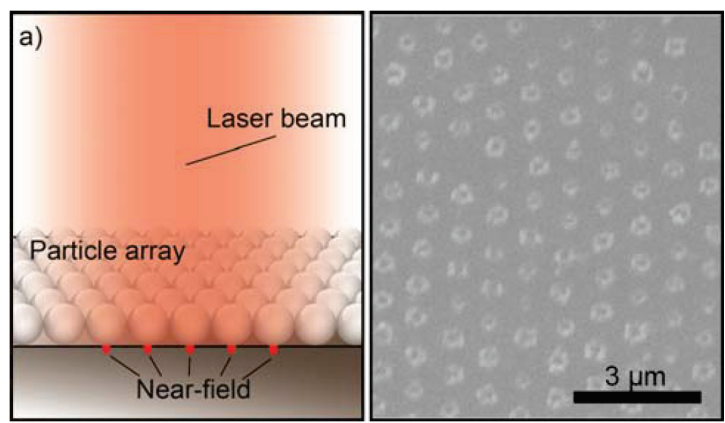

Fig. 2: Principle of CPLA nanostructuring: micro-particles condense in a hexagonal grid and are illuminated with pulsed laser radiation (a). Near-field focusing underneath the particles then leads to periodical nano-holes (b).

\subsection{Optical trap assisted nanostructuring}

The disadvantages of these approaches can be overcome by using a tightly focused cw laser beam as an optical trap, in order to move and position single microspheres which again act as micro-lenses. The optical trapping approach offers complete freedom in the geometry of generated features, whereas it is still robust and does not require sophisticated positioning controls. Figure 3 displays the basic principle of optical trap assisted nanostructuring.

An experimental proof of principle has recently been realised by McLeod and Arnold [20]. They used small polystyrene microspheres with a diameter of $d=0.76 \mu \mathrm{m}$ and a laser with a wavelength of $\lambda=355 \mathrm{~nm}$ and pulse durations of $\tau=15 \mathrm{~ns}$ for direct-writing of nanostructures on polymer coated glass substrates. For the positioning of the particles, they applied a Bessel beam optical trap. In opposite to Gaussian optical tweezers, Bessel beam laser traps do not feature gradient forces along the axis of beam propagation [21] and therefore do not exhibit a stable threedimensional fixation of trapped particles. They rather keep particles on the beam axis, where the light scattering leads to a net force in the beam propagation direction.

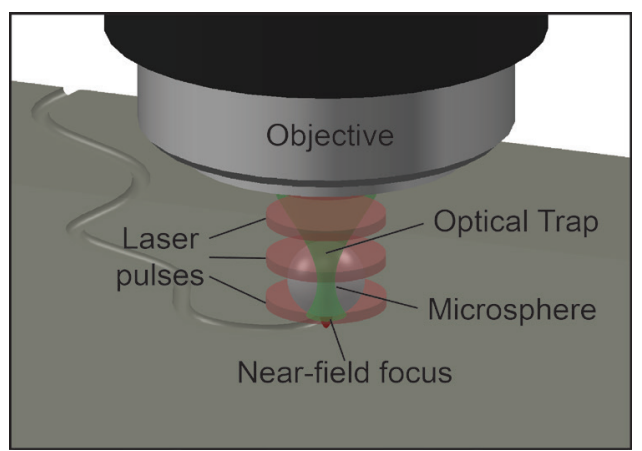

Fig. 3: Principle of optical trap assisted nanostructuring

In case of the previously mentioned CPLA surface structuring, the particles have direct contact to the substrate surface. It is beneficial, however, to maintain a defined distance between particle and substrate to achieve the smallest feature sizes of generated structures [20]. This distance mostly depends on the particle size. In our case $(\mathrm{d}=2 \mu \mathrm{m})$ it is on the order of hundreds of nanometres. In case of Bessel beam optical traps, proper spacing is maintained by counterbalancing the scattering force by electrostatic double-layer repulsion between the microsphere and the substrate surfaces. Since the scattering force is directly depending on the photon flux, the right distance between particle and substrate can be controlled by the trapping laser power alone [20].

However, the stiffness of an optical trap also scales linearly with the applied laser power. Positioning of particles by a Bessel beam optical trap therefore limits the maximal optical forces and the trapping stiffness. In case of laser ablation of the substrate underneath the trapped particle, the microsphere will be affected by the ablation process. Material gets accelerated in the direction of the particle with every laser pulse and thereby leads to displacement of the bead, or at higher pulse energies may even result in the loss of the trapped particle [22].

Depending on the scale of this displacement, it can take a significant time until the particle is brought back to its equilibrium by the optical forces. This obviously limits the usable pulse repetition rate and therefore slows down the entire structuring process.

To overcome this limitation, we propose the use of Gaussian optical tweezers for the positioning of the particles. By using Gaussian tweezers instead of a Bessel beam trap, the particles are confined in all three dimensions so that it is possible to apply higher laser powers to increase the trapping stiffness. This leads to an increase in the maximal pulse frequency and thus to a faster structuring process. The distance between surface and particle, however, has to be controlled by moving the substrate relative to the trapping laser beam.

\section{Experimental setup and procedure}

Our experimental setup is designed like an inverse optical microscope. It consists of optical and opto- 
mechanical off-the-shelf parts and combines three beam paths: the trapping laser, the pulsed structuring laser and the imaging system. The optical trap is realised by a cw Nd:YAG laser $(\lambda=1064 \mathrm{~nm})$ with high beam quality $\left(\mathrm{M}^{2}<1.1\right)$ and an output power up to $5 \mathrm{~W}$. Its beam is magnified by two lenses before it is focused by an Olympus water immersion objective with a numerical aperture (NA) of 1.2 to create a stable three-dimensional Gaussian optical trap. After the focal plane the trapping beam is reimaged onto a quadrant photodiode, which can be used to measure the forces on a trapped particle and the trap stiffness. The sample is located in the focal plane of the objective and positioned with a three-dimensional stage with stepper motors and piezo actuators (Thorlabs NanoMax 311).

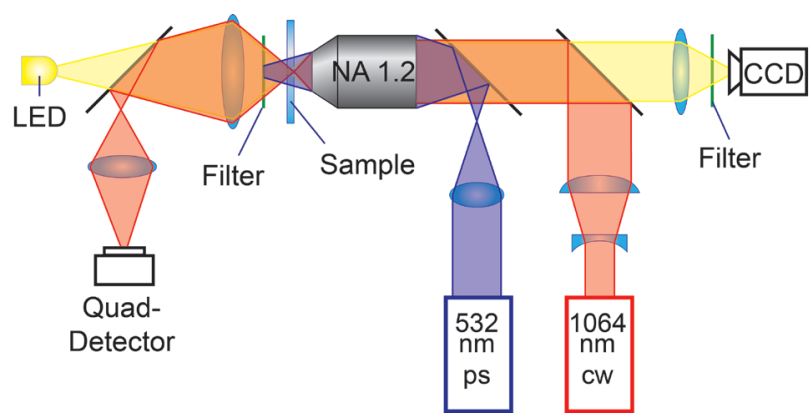

Fig. 4: The experimental setup combines three beam paths: Trapping laser (red), pulsed structuring laser (blue) and imaging system (yellow)

For structuring, we used a picosecond laser with wavelength $\lambda=532 \mathrm{~nm}$, pulse duration $\tau=10 \mathrm{ps}$ and a pulse repetition rate from single shot to $8.2 \mathrm{MHz}$ (Time Bandwidth Fuego). The structuring laser beam is demagnified and a significant divergence of the beam is created. This is necessary in order not to focus the pulsed laser into the objective focal plane and onto the trapped particle, but rather to illuminate the particle as homogeneously as possible. To observe the structuring process, the substrate plane is illuminated by a white light LED and imaged onto a CCD camera. A schematic of the experimental setup is displayed in Fig. 4.

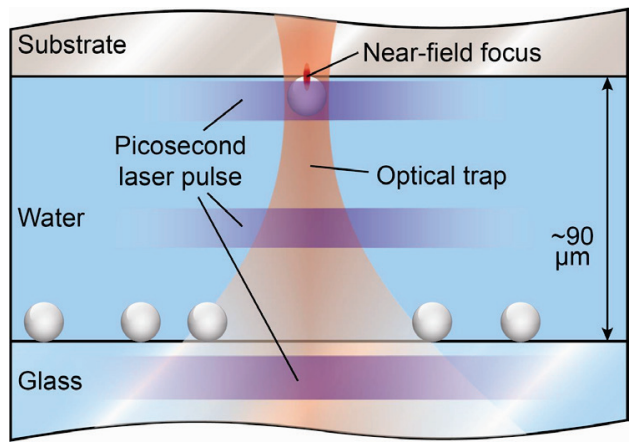

Fig. 5: Sample layout

The samples consist of soda lime glass coated with thin films of polyimide with a thickness of $\sim 150 \mathrm{~nm}$. A thin aquatic solution of microspheres is deposited onto a substrate and sealed with double sided tape and a microscopy cover slide. The volume which is formed by the tape has a thickness of about $90 \mu \mathrm{m}$. The particles, in this case $2 \mu \mathrm{m} \mathrm{SiO}{ }_{2}-$ microspheres, are trapped in the focus of the cw Nd:YAG laser. By moving the whole sample, the sphere is positioned near the upper surface, which is the polyimide coated glass. The picosecond laser illuminates the particle with a fluence slightly below the ablation threshold of the substrate. Focusing by the microsphere leads to the generation of nanometre scale structures. Figure 5 displays a schematic of the sample.

\section{Nanostructuring results}

With our experimental setup, it was possible to demonstrate Gaussian optical trap assisted nanostructuring. For the first experiments we used relatively large $(\mathrm{d}=2 \mu \mathrm{m}) \mathrm{SiO}_{2}$ particles, in order to make the process easily observable on a CCD camera. Although it is very prone to small misalignments in the optical beam paths, the nanostructuring process turned out to very robust and reproducible.

To achieve high position accuracy while structuring, a very stiff optical trap is needed. As mentioned before, the stiffness can be increased by increasing the trapping laser power. However, in case of substrate materials with low damage threshold, the risk of damaging the surface by the tightly focused trapping laser limits its maximal usable power. We found that trapping with up to $500 \mathrm{~mW}$ $(\lambda=1064 \mathrm{~nm})$ was possible without altering the substrate.

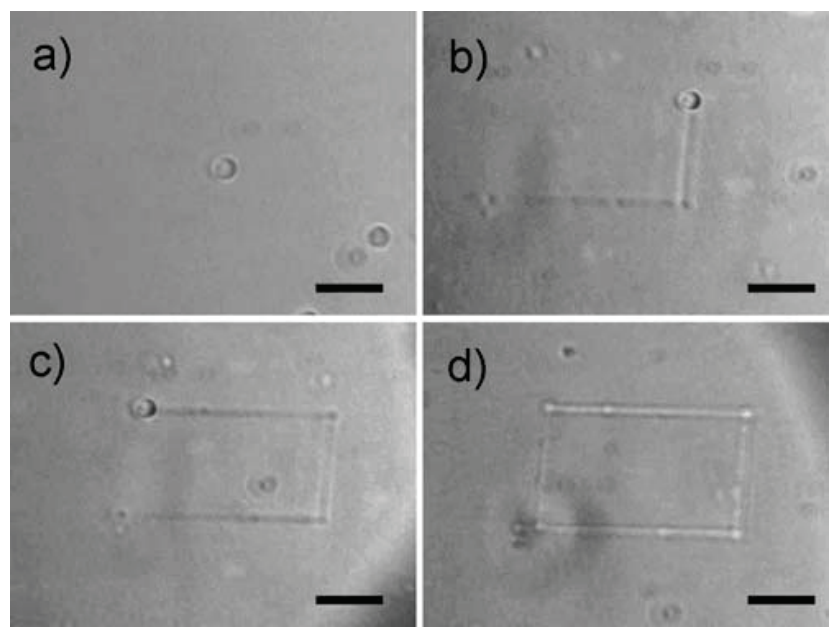

Fig. 6: Video sequence of the nanostructuring process: positioning of the particle near the surface (a), structuring with a high feed rate of $v=20 \mu \mathrm{m} / \mathrm{s}(\mathrm{b}, \mathrm{c})$, nanostructured surface (d). All scale bars are $5 \mu \mathrm{m}$.

With too high pulse energies of the structuring picosecond laser it is still possible to displace the particle from its equilibrium and thereby to disrupt the structuring process. But with proper spacing of the particle to the surface, the focussing effect of the trapped bead allows ablation with fluences as low as $10 \mathrm{~mJ} / \mathrm{cm}^{2}$. The distance between particle and surface in this case is approximately $500 \mathrm{~nm}$. To set this separation, the trapped particle is positioned directly on the surface and then moved to the desired distance by the piezo stage. To assure that the particle is on the surface, its diffraction pattern can be compared to a particle that is stuck on the surface. When the diffraction rings have the same diameter, two particles are in the same plane. This can be analyzed by image processing software, such as MATLAB. 
In order to achieve short process times, a high pulse repetition rate is necessary to increase the feed rate. However, an increase in pulse frequency can also lead to a displacement of the trapped microsphere, which again results in an unstable structuring process. In our experiments, it was not possible to repeatably generate surface structures with a pulse repetition rate above $10,000 \mathrm{~Hz}$ at a feed rate of $20 \mu \mathrm{m} / \mathrm{s}$. Due to the resulting pulse overlap, the particle is affected by the ablation of several pulses and the effects of all shots accumulate. The overlap could be decreased by a further increase in feed rate, but a faster movement leads to a lateral displacement of the particle with regard to the optical trap because of Stokes' friction in the aqueous solution.

However, laser nanostructuring with Gaussian optical tweezers still offers the possibility of generating nanoscale features at high feed rates. At a rate of $20 \mu \mathrm{m} / \mathrm{s}$ we were able to produce defined line structures with a minimum line width of $350 \mathrm{~nm}$ (edge-to-edge) with use of $2 \mu \mathrm{m}$ particles. Lower feed rates of $5 \mu \mathrm{m} / \mathrm{s}$ allow for deep $(\sim 150 \mathrm{~nm})$, more pronounced structures at the cost of a broader line width of $500 \mathrm{~nm}$ as displayed in Fig. 7.

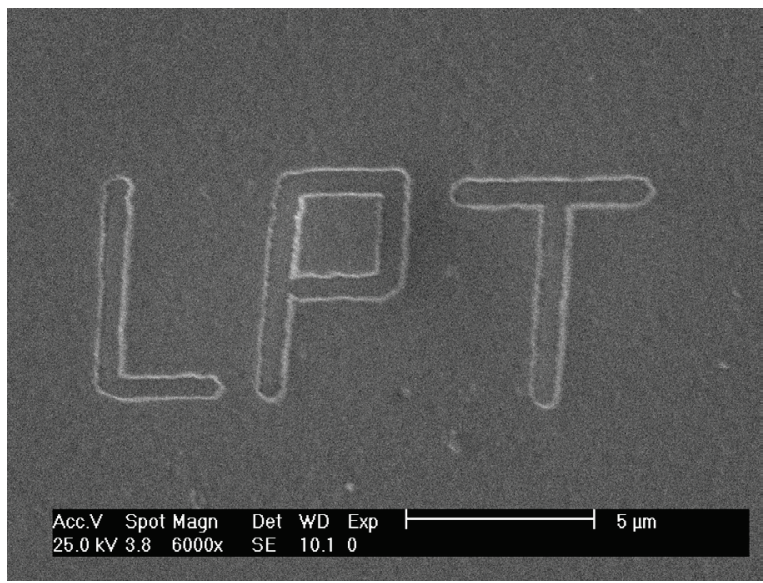

Fig. 7: SEM-microphotograph of nanostructures on polyimide (EPO-Tek P1011). The particle used for focusing is a $2 \mu \mathrm{m} \mathrm{SiO}_{2}$ microsphere positioned with Gaussian optical tweezers $(\lambda=1064 \mathrm{~nm})$. To induce the structuring process the particle is illuminated by a $\mathrm{Nd}: \mathrm{YVO}_{4}$ picosecond laser with $\lambda=532 \mathrm{~nm}$, $\tau=10 \mathrm{ps}$, pulse frequency $\mathrm{f}=500 \mathrm{~Hz}$ and fluence $\mathrm{F}=8 \mathrm{~mJ} / \mathrm{cm}^{2}$.

\section{Conclusion and outlook}

Our experiments showed that direct-writing of submicrometer-structures is possible by positioning of microspheres with Gaussian optical tweezers and illumination with pulsed laser radiation. The application of Gaussian optical traps allow high feed rates up to $20 \mu \mathrm{m} / \mathrm{s}$ when generating nanostructures with $2 \mu \mathrm{m} \mathrm{SiO}{ }_{2}$ beads on polyimide coated glass samples. The minimum line width of repeatable generated structures is $350 \mathrm{~nm}$.

A decrease in feature size is possible by decreasing the particle size and furthermore by the use of femtosecond instead of picosecond laser pulses. The stiffness of the optical trap does not decrease for smaller particles and therefore it should be possible to maintain the high feed rates.

\section{Acknowledgments}

The authors gratefully acknowledge funding of the project within the priority programme 1327 "Optisch erzeugte Sub-100-nm Strukturen für biomedizinische und technische Applikationen" and the funding of the Erlangen Graduate School in Advanced Optical Technologies (SAOT) by the German National Science Foundation (DFG).

\section{References}

[1] I. Etsion: J. Tribol, 127, 1, 2005, p. 248.

[2] A. Kurella: Journal of Biomaterials Applications, 20, 1, 2005, pp. 5-50.

[3] V. Kohli and A.Y. Elezzabi: WIREs Nanmed. Nanobiotech., 1, 1, 2009, p. 11-25.

[4] E. Abbe: Archiv f. mikroskop. Anatomie, 9, 1, 1873, p. 413-418.

[5] S.R.S. Rayleigh: Philosophical Magazine, 5, 1896, p. 29.

[6] K.-S. Lee, D.-Y. Yang, S.H. Park and R.H. Kim: Polym. Adv. Technol., 17, 2, 2006, pp. 72-82.

[7] L. Novotny and B. Hecht: Principles of Nano-Optics. Cambridge: Cambridge University Press, 2006.

[8] D. Pohl, W. Denk and M. Lanz: Applied Physics Letters, 44, 7, 1984, p. 3.

[9] A. Lewis, M. Isaacson, A. Harootunian and A. Muray: Ultramicroscopy, 13, 3, 1984, p. 5.

[10] E. Betzig, J. Trautman, R. Wolfe, E. Gyorgy, P. Finn, M. Kryder and C.-H. Chang: Applied Physics Letters, 61, 2, 1992, p. 3.

[11] B. Hecht, H. Bielefeldt, L. Novotny, Y. Inouye and D. Pohl: Phys. Rev. Lett., 77, 9, 1996, p. 4.

[12] K. Dickmann, J. Jersch and F. Demming: Surface and Interface Analysis, 25, 7-8, 1997, p. 5.

[13] Y. Lu, Z. Mai, Y. Zheng and W. Song: Appl. Phys. Lett., 76, 9, 2000, p. 3.

[14] Z. Wang, N. Joseph, L. Li and B. Luk'yanchuk: Proceedings of the Institution of Mechanical Engineers, Part C, 224, 5, 2009, p. 15.

[15] H.-J. Münzer, M. Mosbacher, M. Bertsch, J. Zimmermann, P.O. Leiderer and J. Boneberg: Journal of Microscopy, 202, 1, 2000, p. 7.

[16] K. Vestentoft, J. Oelsen, B. Christensen and P. Balling: Nanostructuring of surfaces by ultra-short laser pulses, 80, 3, 2005, p. 4.

[17] N. Nedyalkov, H. Takada and M. Obara: Applied Physics A, 85, 2006, p. 6.

[18] Z. Zhou, M. Hong, J.Y. Fuh, L. Lu, B. Luk'yanchuk, Z. Wang, L. Shi and T. Chong: Appl. Phys. Lett., 88, 2, 2006, p. 3 .

[19] K.-H. Leitz, U. Quentin, B. Hornung, A. Otto, I. Alexeev and M. Schmidt: Proceedings of SPIE, 7925, 2011, p. 15.

[20] E. McLeod and C.B. Arnold: Nature Nanotechnology, 3, 2008, p. 12.

[21] R. Fardel, E. McLeod. Y.-C. Tsai and C. B. Arnold. Appl. Phys. A, 101, 2010, pp. 41-46.

[22] D.G. Grier: Nature, 424, 6950, 2003, p. 810-816. 